\title{
Insulin Receptor-Associated Roles of Serotonin Transporter
}

\author{
Fusun Kilic ${ }^{1 *}$ \\ Department of Biology, Merced College, USA
}

*Corresponding author: Fusun Kilic, Department of Biology, Merced College, Merced, California, USA

To Cite This Article: Fusun Kilic, Insulin Receptor-Associated Roles of Serotonin Transporter. Am J Biomed Sci \& Res. 2021 - 12(1). AJBSR. MS.ID.001714. DOI: 10.34297/AJBSR.2021.12.001714

Received: 制 January 25, 2021; Published: 阱 February 22, 2021

\begin{abstract}
Insulin signalling plays a major role in maturation and the translocation of serotonin transporter protein (SERT) to the plasma membrane through acting on endoplasmic reticulum (ER) proteins, specifically ERp44. In supporting these reports, SERT was found as arrested at intracellular compartments in diabetes associated placental cells.

Conversely, SERT was described as a regulator of glucose homeostasis. In exploring this feature, the phosphorylation of insulin receptor (IR) was studied in SERT gene knockout (KO) mice and the IR phosphorylation was found significantly downregulated only in the placenta of SERTKO mice. The impact of SERT on the phosphorylation of IR was through their physical association on the plasma membrane which facilitated the phosphorylation of IR and protected the placental cells against apoptosis.
\end{abstract}

Here, this review collated all the known and reported findings and drew a molecular link between SERT and insulin receptor.

\section{Introduction}

\section{Insulin signaling and SERT}

The serotonin transporter (SERT; SLC6A4) regulates the concentration and duration of serotonin (5-HT) at extracellular compartments via a saturable re-uptake mechanism [1]. However, this well-defined transporter role of SERT is only one of the others because SERT is a multifunctional plasma membrane protein as protecting cells against apoptosis [2] and a regulator of glucose homeostasis $[3,4]$.

Post-translational modifications play important roles in folding correctly and assembly of the proteins which are leading factors in the efficiency of their functions [5]. As a membrane-bound protein, SERT depends on its correctly folding and cellular location which are regulated by post-translational modifications, such as glycosylation [6-8], disulfide bond formation $[9,10]$ and oligomerizations [11]. These modifications provide SERT monomers a proper folding, structure, membrane trafficking, association with other proteins [12] and assembly on the plasma membrane [13]. Following these modifications SERT monomers are associated in an oligomeric form [11]. In short, SERT is an oligomeric glycoprotein and insulin signaling plays a major role in the maturation, correctly folding and trafficking to the plasma membrane of SERT $[3,10,13]$.
Conformational maturation of membrane proteins involves different pathways for post-translational modifications. Formation of intra or intermolecular disulfide bonds is one of the major rate limiting factors. SERT has three cysteine (Cys) residues on external loop. Disulfide bond formation between two residues, C200 and C209, is required for SERT folding, surface expression, and transport activity $[8,10]$. Disruption of the disulfide bond by a single mutation of C200 or C209 produces mutants with a terminally exposed thiol that are retained intracellularly $[9,10]$. However, mutation of both Cys in the pair allows SERT to reach the plasma membrane but compromises transport activity [14]. Previous studies highlighting the importance of a disulfide bond for SERT folding and surface expression suggest a thiol-dependent quality control mechanism in SERT maturation $[9,14]$. Moreover, the modification of exposed thiols with $\beta$-mercaptoethanol (MSH) altered the density of SERT molecules on the plasma membrane and the 5-HT uptake rates [10]. Pretreatment of the cells with MSH led to much lower 5-HT uptake rates when compared to control cells even though MSHtreatment released more SERT molecules to the plasma membrane. The association between SERT molecules also was obstructed by MSH treatment $[8,10]$. All together, these studies indicated the involvement of SERT in thiol-mediated retention mechanism [8] 
but more importantly disulfide bond formation maintained the association of SERT monomers in an oligomeric form [10].

The roles of two endoplasmic reticulum chaperones, ERp44 and ER Oxidase1- $\alpha$ (Ero1-L $\alpha$ ) are well defined in the maturation and quality control of disulfide containing oligomeric proteins [15$17,23]$. These two thioredoxin family members, ER chaperones first bind to the exposed thiol on the protein and form mixed disulfide bonds [17-23]. During this process, ERp44 preferentially associates with unassembled subunits of disulfide containing oligomeric proteins $[15,20,21]$. Various biochemical and molecular biological techniques assess an association between ERp44 and SERT, the role of Ero1-L $\alpha$ in the maturation process, and the corresponding impacts on SERT localization and function at the plasma membrane. ERp44 silenced cells led to increased levels of SERT at the cell surface. The functional state of SERT was also compromised based on changes in the mechanism and maximal rate of 5-HT uptake by these cells. Moreover, SERT mutants (C200S, C209S and C109A) with compromised disulfide bond formation and hence structure and function were shown to preferentially associate with ERp44. These studies suggest the importance of disulfide bond formation as a critical step in SERT folding to a fully active form. Also, demonstrated that SERT utilizes ERp44 and Ero1-L $\alpha$, an oxidoreductase in disulfide bond formation process [15,20,21].

These findings from in vitro study models were evaluated in clinical and preclinical study models [8]. In placentas from the diabetes-associated pregnancy, SERT was located with ER $[8,10]$. Furthermore, ERp44 was bound to Cys200/Cys209 on SERT [24]. In nondiabetic placenta, SERT was dissociated from ERp44 only after insulin treatment which allows the transporter proteins to be translocated to the plasma membrane [24]. However, in diabetic condition, under the lack of insulin signaling ERp44 was associated with Cys200/Cys209 on SERT [8,24,25]. Consequently, in placentas from the diabetes-associated pregnancy, the glycolytic enzymes could not modify the N-glycosylation sites, Asp208 which is buried between the occupied Cys200 and Cys209 on SERT $[7,8,10]$. Therefore, through two negative feedback mechanisms ERp44 links to SERT in the placentas of diabetes-associated pregnancy. Based on these findings, it was proposed that post-translational modifications of SERT in placental cells are impaired due to the lack of insulin signaling in diabetes-associated pregnancy [8]. These studies demonstrated that insulin signaling is the major mediator in glycosylation, disulfide bond formation and oligomerization of SERT. In diabetes, due to defective insulin signaling, SERT cannot fold properly during the post-translational modifications. These highlighted studies emphasized the impact of insulin signaling to the modification of SERT.

\section{SERT, a regulator of glucose homeostasis}

Upon binding to insulin, the insulin receptor (IR) is phosphorylated and instantly activates intracellular signaling and under diabetic conditions the phosphorylation of IR is impaired. Insulin signaling is balanced between the phosphorylation and dephosphorylation of IR by tyrosine kinases and phosphatases. A non-responsive IR to insulin signaling is the most common metabolic complication of obesity and non-insulin-dependent diabetes mellitus, such as type 2 diabetes and gestational diabetes mellitus (GDM), the most common metabolic complication of pregnancy, affecting up to $10-15 \%$ of all pregnancies $[26,27]$.

In addition to self-association between $\alpha$ - and $\beta$-subunits, IR also associates with other proteins, which stabilize IR at the cell surface and alter the structure of the receptor [25,28,29]. Interestingly a physical association between IR molecules and SERT was reported in placental cells, but not in placentas of diabetic subjects [25]. Furthermore, studies with preclinical models report that mice lacking the gene for SERT (SERT-KO) [30-33] exhibit insulin resistance and glucose intolerance, and progressively develop obesity and hepatic steatosis [34]. These studies clearly throw additional role to SERT in placental cells more than functioning as a transporter, protecting the trophoblast cells against continuous 5-HT signaling.

Based on these and the follow up studies, SERT was determined as regulator of the body fat storage and glucose homeostasis [3,4]. However, this association was altered by insulin treatment as well as by diabetic conditions [3,8]. The association between SERT-IR was reported as a requirement for the phosphorylation of IR and in turn for the insulin signaling [25]. In SERT gene silenced, SERT-KO mouse placenta the phosphorylation of IR was found significantly reduced [25]. These findings present and strongly support the reported findings [2] on the role of SERT-IR association in insulin signaling via facilitating the phosphorylation of IR in placental cells [2,25].

\section{SERT against in cell death}

SERT, as a transporter plays important roles in health and diseases via regulating the levels of 5-HT in extracellular surface. However, SERT has other roles as a plasma membrane protein. SERT binds IR and facilitates its phosphorylation and regulates the insulin signaling. Further studies explored additional role of SERT in cell death and apoptosis [2].

The histochemical analysis of placentas dissected from 18d pregnant mice lacking the gene encoding SERT showed an abnormal thick band of fibrosis and necrosis [2]. Interestingly, the necrotic insult was primarily located at the junctional zone of the placenta. In continuation, studies found a relationship between the levels of SERT on cell surface with the rate of cell death and presenting an additional role for SERT as protecting placental cells against cell death [2]. The biological features of placentas from SERT-KO mice showed a 49-fold increase in cell death without a concurrent change in the DNA repair or cell proliferation compared to WT counterparts. 
In in vitro settings, the placental choriocarcinoma (JAR) cells demonstrated some, but not all, of the SERT inhibitors caused DNA fragmentation and apoptosis [35]. The findings from these previous studies indicate that SERT on the cell surface was required to prevent programmed cell death. The TUNEL assays located the highest rate of cell death at the maternal side of the placentas in both transgenic mice, but predominantly in the SERT-KO model. This finding is similar to the necrosis described in human placentas complicated by maternal hypertension [36].

In SERT-KO plasma insulin level is fourfold higher and the glucose level is slightly higher than in WT mice. Therefore, the elevated plasma 5-HT in SERT-KO mice is the likely cause of their resistance to elevated plasma insulin. SERT-KO mice develop obesity and cardiovascular complications, and their embryos show various developmental defects [37]. In collating the reported studies with SERT this review article draws a molecular link between SERT and IR in regulating insulin signaling and associated cell death.

\section{References}

1. Rudnick G, Clark J (1993) From synapse to vesicle: the reuptake and storage of biogenic amine neurotransmitters. Biochim Biophys Acta Rev Bioenerg 1144(3): 249-263.

2. Hadden C, Fahmi T, Cooper A, Savenka AV, Lupashin VV, et al. (2017) Serotonin transporter protects the placental cells against apoptosis in caspase 3-independent pathway. J Cell Physiol 232(12): 3520-3529.

3. Unal R, Ahmed BA, Jeffus BA, Harney JT, Lyle CS, et al. (2007) Neurochem 101(4): 937-948.

4. Murphy DL, Lesch KP (2008) Targeting the murine serotonin transporter: insights into human neurobiology. Nat Rev Neurosci 9(2): 85-96.

5. Cooper A, Woulfe DS, Kilic F (2018) Pharmacological Research pii: S1043-S6618(18): 31314-31318.

6. Tate CG, Whiteley E, Betenbaugh MJ (1999) Molecular chaperones stimulate the functional expression of the cocaine-sensitive serotonin transporter. J Biol Chem 274(25): 17551-17558.

7. Ozaslan D, Wang S, Ahmed B, Bene A, Kocabas AM, et al. (2003) Expression of Concern: Glycosyl modification facilitates homo- and hetero-oligomerization of the serotonin transporter: A specific role for sialic acid residues. J Biol Chem 294(13): 5210.

8. Li Y, Hadden C, Singh P, Mercado C, Murphy P, et al. (2014) Proc Natl Acad Sci USA 111: 52.

9. Chen JG, Lui-Ches S, Rudnick G (1997) External Cysteine Residues in the Serotonin Transporter. Biochemistry 36(6): 1479-1486.

10. Freyaldenhoven S, Li Y, Kocabas AM, Ziu E, Ucer S, et al. (2012) The role of ERp44 in maturation of serotonin transporter protein. J Biol Chem 287: 17801-17811.

11. Kilic F, Rudnick G (2000) Oligomerization of serotonin transporter and its functional consequences. PNAS USA 97(7): 3106-3111.

12. Ahmed B, Bukhari IA, Jeffus BC, Harney JT, Thyparambil S, et al. (2009) PLoS One 4: e4730.

13. Mercado C, Kilic F (2010) Molecular Interventions. 10(4): 231-241.

14. Stephan MM, Chen MA, Penado KM, Rudnick G (1997) Biochemistry 36: 1322-1328.

15. Anelli A, Sitia R (2008) "Protein Quality Control in the Early Secretory Pathway”. EMBO 27(2): 315-327.
16. Aridor M, Balch WE. (1996) Membrane fusion: timing is everything. Nature 383(6597): 220-221.

17. Ellgaard L, Helenius A (2003) "Quality control in the endoplasmic reticulum". Nature Rev Mol Cell Biol 4(3): 181-191.

18. Fewell SW, Travers KJ, Weissman JS, Brodsky JL (2001) "The action of molecular chaperones in the early secretory pathway." Annu Rev Genet 35: 149-191.

19. Ellgaard L, Molinari M, Helenius A (1999) "Setting the standards: quality control in the secretory pathway. Science 286(5446): 1882-1888.

20. Anelli T, Alessio M, Bachi A, Bergamelli L, Bertoli G, et al. (2003) “Thiolmediated protein retention in the endoplasmic reticulum: the role of ERp44." EMBO 22(19): 5015-5022.

21. Anelli T, Ceppi S, Bergamelli L, Cortini M, Masciarelli S, et al. (2007) "Sequential steps and checkpoints in the early exocytic compartment during secretory IgM biogenesis." EMBO 26(19): 4177-4188.

22. Kleizen B, Braakman I (2004) "Protein Folding and Quality Control in the Endoplasimic Reticulum." Current Opinion in Cell Biology 16(4): 343349.

23. Otsu M, Sitia R (2007) “Diseases originating from altered protein quality control in the endoplasmic reticulum. Curr Med Chem 14(15): 16391652.

24. Li Y, Cooper A, Odibo IN, Ahmed A, Murphy P, et al. (2016) Discrepancy in Insulin Regulation between Gestational Diabetes Mellitus (GDM) Platelets and Placenta. J Biol Chem 291(18): 9657-9665.

25. Kilic F, Moutkine I, Maroteaux L (2019) Current Topics in Biochemical Research.

26. Herrera R, Rosen OM (1986) Autophosphorylation of the insulin receptor in vitro. Designation of phosphorylation sites and correlation with receptor kinase activation. J Biol Chem 261(26): 11980.

27. Buchanan TA, Xiang A, Kjos SL, Watanabe R (2007) What is gestational diabetes? Diabetes Care 30(Suppl 2): S105-S111.

28. Syed RS, Reid SW, Li C, Cheetham JC, Aoki KH, et al. (1998) Efficiency of signalling through cytokine receptors depends critically on receptor orientation. Nature 395(6701): 511-516.

29. Livnah O, Johnson DL, Stura EA, Farrell FX, Barbone FP, et al. (1998) Nat Struct Biol 5: 993.

30. Uceyler N, Schutt M, Palm F, Vogel C, Meier M, et al. (2010) Lack of the serotonin transporter in mice reduces locomotor activity and leads to gender-dependent late onset obesity. Int J Obes (Lond) 34(4): 701-711.

31. Chen X, Margolis KJ, Gershon MD, Schwartz GJ, Sze JY (2012) Reduced serotonin reuptake transporter (SERT) function causes insulin resistance and hepatic steatosis independent of food intake. PLoS One 7(3): e32511.

32. Bengel D, Murphy DL, Andrews AM, Wichems CH, Feltner D, et al (1998a) Mol Pharmacol 53: 649-655.

33. Walther DJ, Peter JU, Winter S, Holtje M, Paulmann N, et al. (2003) Serotonylation of small GTPases is a signal transduction pathway that triggers platelet alpha-granule release. Cell 115(7): 851-862.

34. Armitage JA, Poston L, Taylor PD (2008) Developmental origins of obesity and the metabolic syndrome: the role of maternal obesity. Front Horm Res 36: 73-84.

35. Bengel D, Isaacs KR, Heils A, Lesch KP, Murphy DL (1998b) Neuroreport 9: 2989-2993.

36. Odibo AO, Patel KR, Spitalnik A, Odibo L, Huettner P (2014) Placental pathology, first-trimester biomarkers and adverse pregnancy outcomes. J Perinatol 34(3): 186-191.

37. Cote F, Fligny C, Bayard E, Launay JM, Gershon MD, et al. (2007) Maternal serotonin is crucial for murine embryonic development. Proc Natl Acad Sci USA 104(1): 329-334 